\title{
DISCRETE MODELING OF THE THREE SPECIES SYN-ECOSYSTEM WITH UNLIMITED RESOURCES
}

\section{B. Hari Prasad}

Department of Mathematics, Chaitanya Degree and PG College (Autonomous) Hanamkonda, Telangana State, India-506 001

e-mail: sumathi_prasad73@yahoo.com

\begin{abstract}
In this paper, the three species syn-ecosystem is comprised of a commensal $\left(\mathrm{S}_{1}\right)$, two hosts $S_{2}$ and $S_{3}$, i.e. $S_{2}$ and $S_{3}$ both benefit $S_{1}$ without getting themselves affected either positively or adversely. Further, $S_{2}$ is a commensal of $S_{3}, S_{3}$ is a host of both $S_{1}, S_{2}$ and all the three species have unlimited resources. The basic equations for this model constitute as three first order non-linear coupled ordinary difference equations. All possible equilibrium states are identified based on the model equations at two stages and criteria for their stability are discussed. Further, the numerical solutions are computed for specific values of the various parameters and the initial conditions.
\end{abstract}

Keywords: commensal, equilibrium state, host, stable, unstable

2010 Mathematics Subject Classification: 92D25, 92D40

\section{Introduction}

Ecology, a branch of evolutionary biology, deals with living species that coexist in a physical environment and sustain themselves on common resources. It is a common observation that the species of the same nature can not flourish in isolation without any interaction with species of different kinds. Syn-ecology is an ecosystem comprised of two or more distinct species. Species interact with each other in one way or other. The ecological interactions can be broadly classified as ammensalism, competition, commensalism, neutralism, mutualism, predation, parasitism and so on. Lotka [1], Svirezhev and Logofet [2] and Volterra [3] pioneered theoretical ecology significantly and opened new eras in the field of life and biological sciences. The authors Rogers and Hassell [4], Varma [5] and Veilleux [6] discussed prey-predator, competing ecological models. Colinvaux [7] and Smith [8] studied basic concepts of population models in ecology.

Mathematical modeling plays a vital role in providing insight into the mutual relationships (positive, negative) between the interacting species. The general concepts of modeling have been discussed by several authors: Kapur [9], Kushing [10], 
Meyer [11] and Pielou [12]. Srinivas [13] studied the competitive ecosystem of two species and three species with limited and unlimited resources. Later, Narayan and Pattabhiramacharyulu [14] studied prey-predator ecological models with partial cover for the prey and alternate food for the predator. Acharyulu and Pattabhiramacharyulu $[15,16]$ derived some productive results on various mathematical models of ecological ammensalism with multifarious resources in the manifold directions. Further, Kumar [17] studied some mathematical models of ecological commensalism. The present author Prasad [18-21] investigated continuous and discrete models on the three species syn-ecosystems.

The present investigation is a discrete model of three species $\left(\mathrm{S}_{1}, \mathrm{~S}_{2}, \mathrm{~S}_{3}\right)$ syn-ecosystem with unlimited resources. The system is comprised of a commensal $\left(\mathrm{S}_{1}\right)$, two hosts $S_{2}$ and $S_{3}$. Further, $S_{2}$ is a commensal of $S_{3}, S_{3}$ is a host of both $S_{1}$ and $S_{2}$.

Commensalism is a symbiotic interaction between two populations where one population $\left(\mathrm{S}_{1}\right)$ gets benefit from $\left(\mathrm{S}_{2}\right)$, while the other $\left(\mathrm{S}_{2}\right)$ is neither harmed nor benefited due to the interaction with $\left(\mathrm{S}_{1}\right)$. The benefited species $\left(\mathrm{S}_{1}\right)$ is called the commensal and the other $\left(\mathrm{S}_{2}\right)$ is called the host. Some real-life examples of commensalism are presented below.

i. The clownfish shelters among the tentacles of the sea anemone, while the sea anemone is not affected.

ii. A squirrel in an oak tree gets a place to live and food for its survival, while the tree remains neither benefited nor harmed.

iii. A flatworm attached to the horse crab and eating the crab's food, while the crab is not put to any disadvantage.

\section{Basic equations of the model}

\subsection{Notation adopted}

$N_{i}(t)$ - the population strength of $\mathrm{S}_{i}$ at time $t, i=1,2,3$

$t \quad$ - time instant

$a_{i} \quad$ - natural growth rate of $\mathrm{S}_{i}, i=1,2,3$

$a_{12}, a_{13}$ - interaction coefficients of $\mathrm{S}_{1}$ due to $\mathrm{S}_{2}$ and $\mathrm{S}_{1}$ due to $\mathrm{S}_{3}$

$a_{23} \quad$ - interaction coefficient of $\mathrm{S}_{2}$ due to $\mathrm{S}_{3}$

Further, the model parameters $a_{1}, a_{2}, a_{3}, a_{12}, a_{13}, a_{23}$ are assumed to be non-negative constants.

\subsection{Basic equations}

Consider the growth of the species during the time interval $(t, t+1)$.

(i) Equation for the first species $\left(N_{1}\right)$ :

$$
N_{1}(t+1)=N_{1}(t)+a_{1} N_{1}(t)+a_{12} N_{1}(t) N_{2}(t)+a_{13} N_{1}(t) N_{3}(t)
$$


(ii) Equation for the second species $\left(N_{2}\right)$ :

$$
N_{2}(t+1)=N_{2}(t)+a_{2} N_{2}(t)+a_{23} N_{2}(t) N_{3}(t)
$$

(iii) Equation for the third species $\left(N_{3}\right)$ :

$$
N_{3}(t+1)=N_{3}(t)+a_{3} N_{3}(t)
$$

\subsection{Species-growth equations in the discrete form}

Consider the nonlinear autonomous system of discrete equations

$$
\begin{gathered}
N_{1}(t+1)=\alpha_{1} N_{1}(t)+a_{12} N_{1}(t) N_{2}(t)+a_{13} N_{1}(t) N_{3}(t) \\
N_{2}(t+1)=\alpha_{2} N_{2}(t)+a_{23} N_{2}(t) N_{3}(t) \\
N_{3}(t+1)=\alpha_{3} N_{3}(t)
\end{gathered}
$$

where

$$
\alpha_{i}=a_{i}+1, i=1,2,3
$$

\section{Equilibrium states}

For a continuous model the equilibrium states are defined by $\frac{d N_{i}}{d t}=0, i=1,2,3$, the equilibrium states for a discrete model are defined in terms of the period of no growth, i.e. $N_{i}(t+r)=N_{i}(t), r=1,2,3, \ldots$, where $r$ is the period of the equilibrium state.

\subsection{One period equilibrium states (Stage-I)}

$$
N_{i}(t+1)=N_{i}(t), i=1,2,3
$$

The system under investigation has only one equilibrium state given by

$$
E_{0}: \bar{N}_{1}=0, \bar{N}_{2}=0, \bar{N}_{3}=0 \quad \text { (Fully washed out state) }
$$


3.1.1. The stability of equilibrium state $E_{0}(0,0,0)$

$$
\begin{aligned}
N_{1}(t)=N_{1}(t+1)= & N_{1}(t+2)=\ldots=0 ; N_{2}(t)=N_{2}(t+1)=N_{2}(t+2)=\ldots=0 ; \\
& N_{3}(t)=N_{3}(t+1)=N_{3}(t+2)=\ldots=0
\end{aligned}
$$

i.e. $N_{i}(t+r)=0$, where $r$ is an integer and $i=1,2,3$.

Hence, $E_{0}(0,0,0)$ is stable.

\subsection{Two period equilibrium states (Stage-II)}

$$
N_{i}(t+2)=N_{i}(t), i=1,2,3
$$

The system under investigation has five equilibrium states given by

(i) Fully washed out state

$$
E_{1}: \bar{N}_{1}=0, \bar{N}_{2}=0, \bar{N}_{3}=0 .
$$

(ii) States in which only the second species survives

$$
\begin{aligned}
& E_{2}: \bar{N}_{1}=0, \bar{N}_{2}=\frac{1-\alpha_{2}}{a_{23}}, \bar{N}_{3}=0, \text { when } \alpha_{2}>1 \\
& E_{3}: \bar{N}_{1}=0, \bar{N}_{2}=-\left[\frac{\left(\alpha_{2}+1\right)+\sqrt{\left(\alpha_{2}+1\right)\left(\alpha_{2}-3\right)}}{2 a_{23}}\right], \bar{N}_{3}=0, \text { when } \alpha_{2}>3 \\
& E_{4}: \bar{N}_{1}=0, \bar{N}_{2}=\frac{\sqrt{\left(\alpha_{2}+1\right)\left(\alpha_{2}-3\right)}-\left(\alpha_{2}+1\right)}{2 a_{23}}, \bar{N}_{3}=0, \text { when } \alpha_{2}>3 \\
& E_{5}: \bar{N}_{1}=0, \bar{N}_{2}=-\frac{2}{a_{23}}, \bar{N}_{3}=0, \text { when } \alpha_{2}=3
\end{aligned}
$$

The states $E_{3}$ and $E_{4}$ coincide when $\alpha_{2}=3$ and do not exist when $\alpha_{2}<3$.

\subsubsection{The stability of equilibrium states}

The equilibrium state $E_{1}$ is stable. Now we will discuss the stability of all other equilibrium states.

\section{The stability of $E_{2}$ :}

$$
N_{1}(t)=N_{1}(t+1)=N_{1}(t+2)=\ldots=0 ; N_{3}(t)=N_{3}(t+1)=N_{3}(t+2)=\ldots=0
$$


i.e. $N_{i}(t+r)=0$, where $r$ is an integer and $i=1,3$

$N_{2}(t+1)=\frac{\alpha_{2}\left(1-\alpha_{2}\right)}{a_{23}} ; N_{2}(t+2)=\frac{\alpha_{2}^{2}\left(1-\alpha_{2}\right)}{a_{23}} ; N_{2}(t+3)=\frac{\alpha_{2}^{3}\left(1-\alpha_{2}\right)}{a_{23}}$ and so on

i.e. $N_{2}(t+r)=\frac{\alpha_{2}^{r}\left(1-\alpha_{2}\right)}{a_{23}}$, where $r$ is an integer.

Hence, $E_{2}$ is unstable.

\section{The stability of $E_{3}$ :}

$$
N_{1}(t)=N_{1}(t+1)=N_{1}(t+2)=\ldots=0 ; N_{3}(t)=N_{3}(t+1)=N_{3}(t+2)=\ldots=0
$$

i.e. $N_{i}(t+r)=0$, where $r$ is an integer and $i=1,3$

$$
\begin{aligned}
& N_{2}(t+1)=-\alpha_{2}\left[\frac{\left(\alpha_{2}+1\right)+\sqrt{\left(\alpha_{2}+1\right)\left(\alpha_{2}-3\right)}}{2 a_{23}}\right] ; \\
& N_{2}(t+2)=-\alpha_{2}^{2}\left[\frac{\left(\alpha_{2}+1\right)+\sqrt{\left(\alpha_{2}+1\right)\left(\alpha_{2}-3\right)}}{2 a_{23}}\right] ; \\
& N_{2}(t+3)=-\alpha_{2}^{3}\left[\frac{\left(\alpha_{2}+1\right)+\sqrt{\left(\alpha_{2}+1\right)\left(\alpha_{2}-3\right)}}{2 a_{23}}\right] \text { and so on }
\end{aligned}
$$

i.e. $N_{2}(t+r)=-\alpha_{2}^{r}\left[\frac{\left(\alpha_{2}+1\right)+\sqrt{\left(\alpha_{2}+1\right)\left(\alpha_{2}-3\right)}}{2 a_{23}}\right]$, where $r$ is an integer.

Hence, $E_{3}$ is unstable.

\section{The stability of $E_{4}$ :}

$$
N_{1}(t)=N_{1}(t+1)=N_{1}(t+2)=\ldots=0 ; N_{3}(t)=N_{3}(t+1)=N_{3}(t+2)=\ldots=0
$$

i.e. $N_{i}(t+r)=0$, where $r$ is an integer and $i=1,3$

$$
\begin{aligned}
& N_{2}(t+1)=\alpha_{2}\left[\frac{\sqrt{\left(\alpha_{2}+1\right)\left(\alpha_{2}-3\right)}-\left(\alpha_{2}+1\right)}{2 a_{23}}\right] ; \\
& N_{2}(t+2)=\alpha_{2}^{2}\left[\frac{\sqrt{\left(\alpha_{2}+1\right)\left(\alpha_{2}-3\right)}-\left(\alpha_{2}+1\right)}{2 a_{23}}\right] ; \\
& N_{2}(t+3)=\alpha_{2}^{3}\left[\frac{\sqrt{\left(\alpha_{2}+1\right)\left(\alpha_{2}-3\right)}-\left(\alpha_{2}+1\right)}{2 a_{23}}\right] \text { and so on }
\end{aligned}
$$


i.e. $N_{2}(t+r)=\alpha_{2}^{r}\left[\frac{\sqrt{\left(\alpha_{2}+1\right)\left(\alpha_{2}-3\right)}-\left(\alpha_{2}+1\right)}{2 a_{23}}\right]$, where $r$ is an integer.

Hence, $E_{4}$ is unstable.

The stability of $E_{5}$ :

$$
N_{1}(t)=N_{1}(t+1)=N_{1}(t+2)=\ldots=0 ; N_{3}(t)=N_{3}(t+1)=N_{3}(t+2)=\ldots=0
$$

i.e. $N_{i}(t+r)=0$, where $r$ is an integer and $i=1,3$

$N_{2}(t+1)=-\frac{2 \alpha_{2}}{a_{23}} ; N_{2}(t+2)=-\frac{2 \alpha_{2}^{2}}{a_{23}} ; N_{2}(t+3)=-\frac{2 \alpha_{2}^{3}}{a_{23}}$ and so on

i.e. $N_{2}(t+r)=-\frac{2 \alpha_{2}^{r}}{a_{23}}$, where $r$ is an integer.

Hence, $E_{5}$ is unstable.

At this stage, in all five equilibrium states, the fully washed out state $E_{1}$ is stable only and all the remaining are unstable.

\section{Computer simulations}

The numerical simulations of the discrete model equations computed for specific values of the various parameters and the initial conditions. The results are illustrated in Figures 1 to 5.

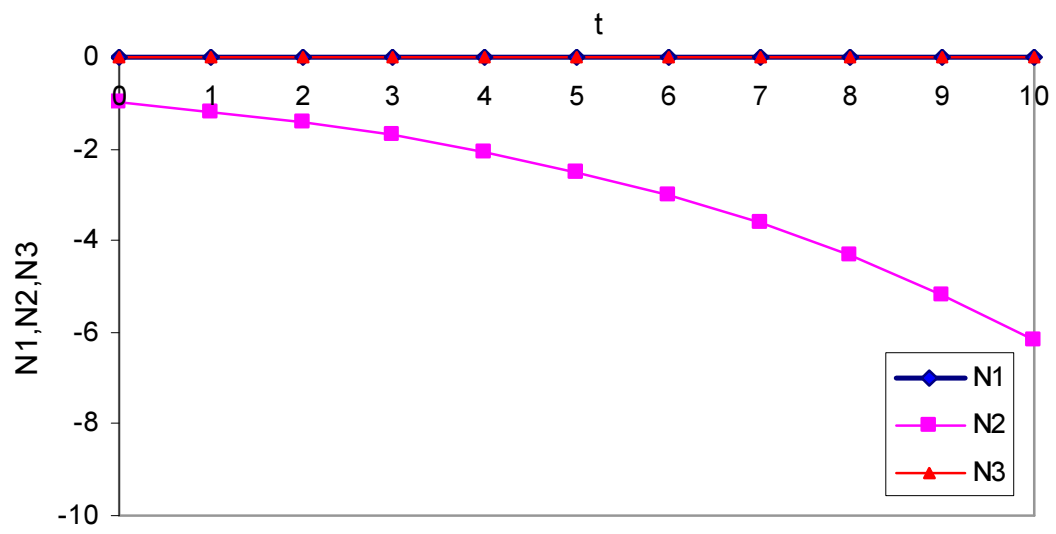

Fig. 1. Variation of $N_{1}, N_{2}$ and $N_{3}$ against time $(t)$ for $\alpha_{1}=1.2, \alpha_{2}=6.9, \alpha_{3}=3.6$, $a_{12}=4.2, a_{13}=5.8, a_{23}=0.2, N_{1}(0)=0, N_{2}(0)=-1, N_{3}(0)=0$ 


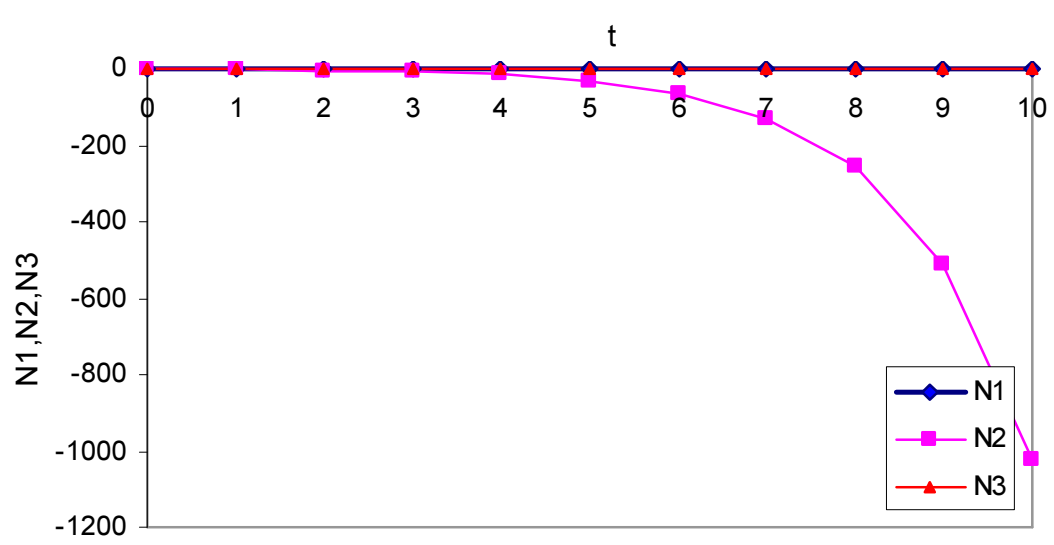

Fig. 2. Variation of $N_{1}, N_{2}$ and $N_{3}$ against time $(t)$ for $\alpha_{1}=5.9, \alpha_{2}=2, \alpha_{3}=2.6$, $a_{12}=1.5, a_{13}=3.8, a_{23}=1, N_{1}(0)=0, N_{2}(0)=-1, N_{3}(0)=0$

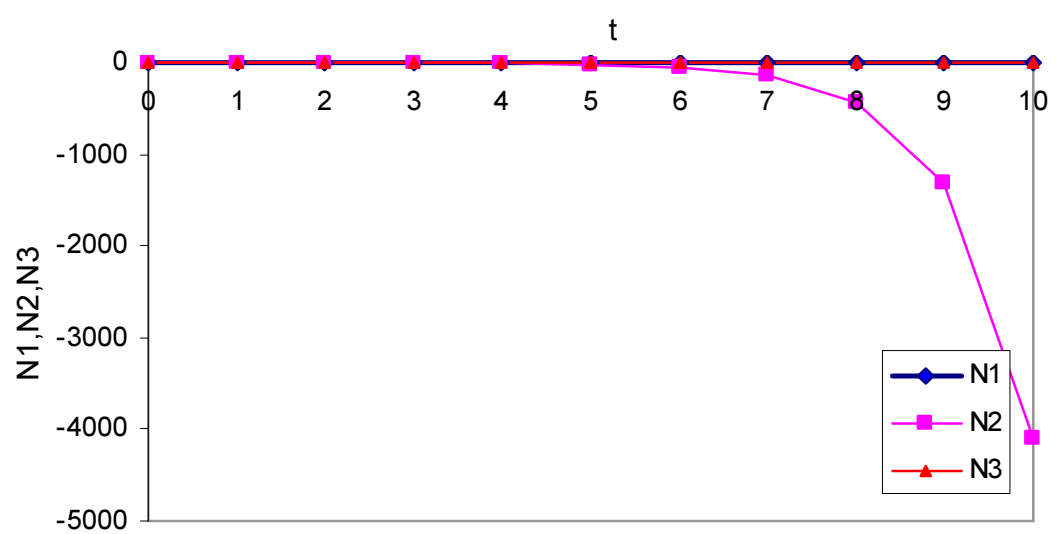

Fig. 3. Variation of $N_{1}, N_{2}$ and $N_{3}$ against time $(t)$ for $\alpha_{1}=3.9, \alpha_{2}=3.1, \alpha_{3}=5$, $a_{12}=1.9, a_{13}=0.2, a_{23}=40, N_{1}(0)=0, N_{2}(0)=-0.05, N_{3}(0)=0$

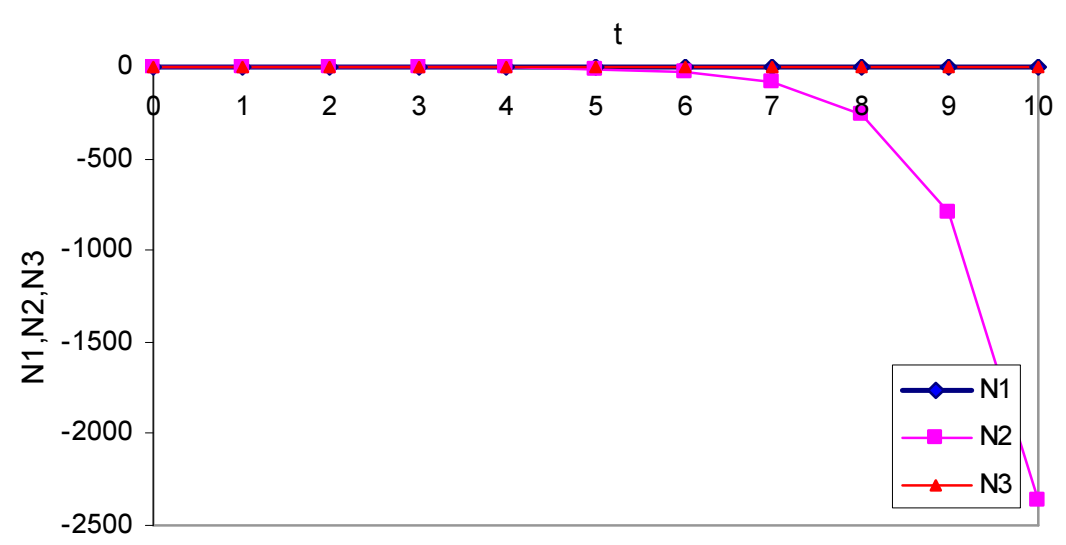

Fig. 4. Variation of $N_{1}, N_{2}$ and $N_{3}$ against time $(t)$ for $\alpha_{1}=0.6, \alpha_{2}=3, \alpha_{3}=5.9$, $a_{12}=2, a_{13}=8.5, a_{23}=48, N_{1}(0)=0, N_{2}(0)=-0.04, N_{3}(0)=0$ 


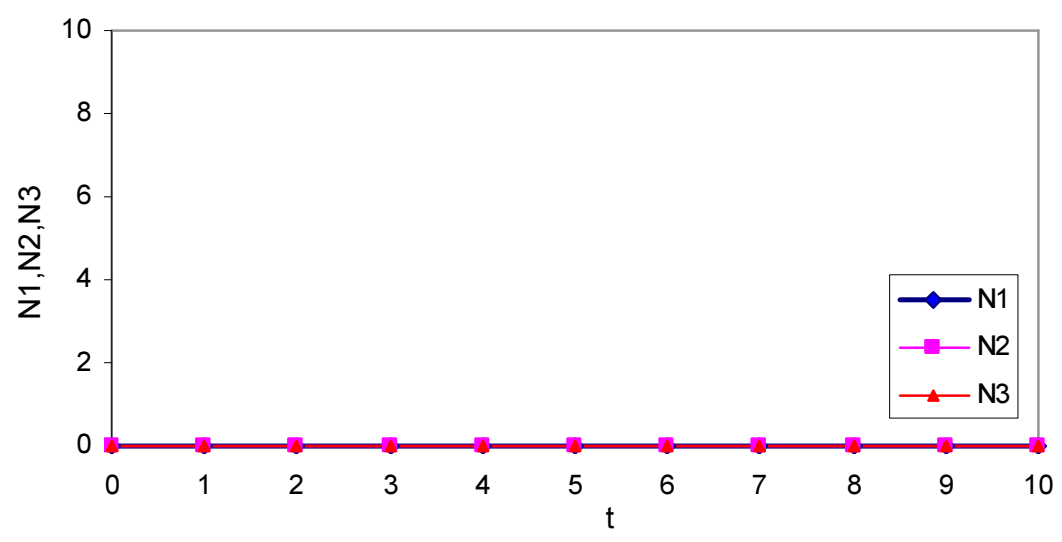

Fig. 5. Variation of $N_{1}, N_{2}$ and $N_{3}$ against time $(t)$ for $\alpha_{1}=9.3, \alpha_{2}=1.3, \alpha_{3}=7.9$, $a_{12}=2.8, a_{13}=3.7, a_{23}=3, N_{1}(0)=0, N_{2}(0)=0, N_{3}(0)=0$

\section{Conclusion}

The present paper deals with an investigation on a discrete model of a three species syn-ecosystem with unlimited resources. The system is comprised of a commensal $\left(S_{1}\right)$ common to two hosts $S_{2}$ and $S_{3}$, i.e. $S_{2}$ and $S_{3}$ both benefit $S_{1}$ without getting themselves affected either positively or adversely. All possible equilibrium points of the model are identified based on the model equations at two stages.

$$
\begin{gathered}
\text { Stage-I : } N_{i}(t+1)=N_{i}(t) ; i=1,2,3 \\
\text { Stage-II : } N_{i}(t+2)=N_{i}(t) ; i=1,2,3
\end{gathered}
$$

In Stage-I there is only one equilibrium point, while in Stage-II there would be five equilibrium points. The equilibrium point $E_{0}$ in Stage-I is found to be stable while in Stage-II only one is stable. Further the numerical solutions for the discrete model equations are computed.

\section{Acknowledgment}

I thank to Professor (Retd) N.Ch. Pattabhi Ramacharyulu, Department of Mathematics, National Institute of Technology, Warangal (T.S.), India for his valuable suggestions and encouragement.

\section{References}

[1] Lotka A.J., Elements of Physical Biology, Williams and Wilking, Baltimore 1925.

[2] Svirezhev Yu.M., Logofet D.O., Stability of Biological Community, MIR, Moscow 1983. 
[3] Volterra V., Leconssen La Theorie Mathematique De La Leitte Pou Lavie, Gauthier-Villars, Paris 1931.

[4] Rogers D.J., Hassell M.P., General models for insect parasite and predator searching behavior, Interference, Journal Anim. Ecol. 1974, 43, 239-253.

[5] Varma V.S., A note on exact solutions for a special prey - predator or competing species system, Bull. Math. Biol. 1977, 39, 619-622.

[6] Veilleux B.G., An analysis of the predatory interaction between Paramecium \& Didinium, Journal Anim. Ecol. 1979, 48, 787-803.

[7] Colinvaux A.P., Ecology, John Wiley, New York 1986.

[8] Smith J.M., Models in Ecology, Cambridge University Press, Cambridge 1974.

[9] Kapur J.N., Mathematical Modeling in Biology \& Medicine, Affiliated East West, 1985.

[10] Kushing J.M., Integro-Differential Equations and Delay Models in Population Dynamics, Lecture Notes in Bio-Mathematics, Springer Verlag, 1977, 20.

[11] Meyer W.J., Concepts of Mathematical Modeling, McGraw-Hill, 1985.

[12] Pielou E.C., Mathematical Ecology, John Wiley and Sons, New York 1977.

[13] Srinivas N.C., Some Mathematical Aspects of Modeling in Bio-medical Sciences, Ph.D Thesis, Kakatiya University, 1991.

[14] Narayan K.L., Pattabhiramacharyulu N.Ch., A prey-predator model with cover for prey and alternate food for the predator and time delay, Int. Journal of Scientific Computing 2007, 1, 7-14.

[15] Acharyulu K.V.L.N., Pattabhiramacharyulu N.Ch., An enemy - ammensal species pair with limited resources - a numerical study, Int. Journal Open Problems Compt. Math. 2010, 3, 339-356.

[16] Acharyulu K.V.L.N., Pattabhiramacharyulu N.Ch., An ammensal-prey with three species ecosystem, International Journal of Computational Cognition 2011, 9, 30-39.

[17] Kumar N.P., Some Mathematical Models of Ecological Commensalism, Ph.D. Thesis, Acharya Nagarjuna University, 2010.

[18] Prasad B.H., On the stability of a three species syn-eco-system with mortality rate for the second species, Int. Journal of Social Science \& Interdisciplinary Research 2014, 3, 35-45.

[19] Prasad B.H., The stability analysis of a three species syn-eco-system with mortality rates, Contemporary Mathematics and Statistics 2014, 2, 76-89.

[20] Prasad B.H., A study on discrete model of three species syn-eco-system with limited resources, Int. Journal Modern Education and Computer Science 2014, 11, 38-44.

[21] Prasad B.H., A discrete model of a typical three species syn-eco-system with unlimited resources for the first and third species, Asian Academic Research Journal of Multidisciplinary 2014, 1, 36-46. 